\title{
Perfect powers in linear recurring sequences
}

\author{
by \\ Clemens Fuchs and Robert F. Tichy (Graz)
}

1. Introduction. Let $A_{1}, \ldots, A_{k}$ and $G_{0}, G_{1}, \ldots, G_{k-1}$ be algebraic numbers over the rationals and let $\left(G_{n}\right)$ be a $k$ th order linear recurring sequence given by

$$
G_{n}=A_{1} G_{n-1}+\ldots+A_{k} G_{n-k} \quad \text { for } n=k, k+1, \ldots
$$

Let $\alpha_{1}, \ldots, \alpha_{t}$ be the distinct roots of the corresponding characteristic polynomial

$$
X^{k}-A_{1} X^{k-1}-\ldots-A_{k} .
$$

Then for $n \geq 0$,

$$
G_{n}=P_{1}(n) \alpha_{1}^{n}+P_{2}(n) \alpha_{2}^{n}+\ldots+P_{t}(n) \alpha_{t}^{n},
$$

where $P_{i}(n)$ is a polynomial with degree less than the multiplicity of $\alpha_{i}$; the coefficients of $P_{i}(n)$ are elements of the field $\mathbb{Q}\left(G_{0}, \ldots, G_{k-1}, A_{1}, \ldots, A_{k}, \alpha_{1}\right.$, $\left.\ldots, \alpha_{t}\right)$.

The recurring sequence is called simple if all characteristic roots are simple. It is called nondegenerate if no quotient $\alpha_{i} / \alpha_{j}$ for all $1 \leq i<j \leq t$ is equal to a root of unity, and degenerate otherwise. Observe that even if $\left(G_{n}\right)$ is degenerate, there exists a positive integer $d$ such that $\left(G_{r+m d}\right)$ is nondegenerate on each of the $d$ arithmetic progressions with $0 \leq r<d$. Therefore, restricting to nondegenerate recurring sequences causes no substantial loss of generality.

In the present paper we deal with the Diophantine equation

$$
G_{n}=E x^{q}, \quad E \in \mathbb{Z} \backslash\{0\},
$$

which was earlier investigated by several authors (e.g. cf. [21]).

For the Fibonacci sequence $\left(F_{n}\right)$, Cohn [2] and Wyler [28] independently proved that $F_{n}$ is a square only if $n=0,1,2$ and 13. Cohn [3] and Steiner

2000 Mathematics Subject Classification: Primary 11D45; Secondary 11B37.

This work was supported by the Austrian Science Foundation FWF, grant S8307MAT. 
[24] solved the equations $F_{n}=2 x^{2}$ and $F_{n}=3 x^{2}$. They also proved the corresponding results for the Lucas sequence $\left(L_{n}\right)$. London and Finkelstein [10] determined all cubes in the Fibonacci sequence; Lagarias and Weisser [9] gave another proof. Steiner [23] derived some partial results for higher powers. The proofs of these results do not depend on estimates for linear forms in logarithms. Pethő [14], [15] used the theory of linear forms in logarithms and computer calculations to determine all the cubes and fifth powers in the Fibonacci sequence.

For a nondegenerate recurring sequence $\left(G_{n}\right)$ of order 2 induced by a (rational) integral recurrence, it has been proved independently by Pethö [13] and Shorey and Stewart [19] that for the solutions $x \in \mathbb{Z},|x|>1$ and $q \geq 2$ of $(3), \max (|x|, q, n)$ is bounded by an effectively computable constant depending only on $E$ and on the sequence $\left(G_{n}\right)$. In fact, Pethö proved that $\max (|x|, q, n)$ is bounded by an effectively computable number depending only on the greatest prime divisor of $E$ and on the coefficients and initial values of $\left(G_{n}\right)$ (provided that the coefficients are coprime integers). Pethö extended this result to the equation $G_{n}=b x^{q}$ with $b \in S$, where $S$ is a set of integers composed solely of a finite number of primes.

Shorey and Stewart [19] proved the above finiteness result for certain recurring sequences of order $>2$. Let $\left(G_{n}\right)$ be a nondegenerate linear recurring sequence given by

$$
G_{n}=\lambda_{1} \alpha_{1}^{n}+P_{2}(n) \alpha_{2}^{n}+\ldots+P_{t}(n) \alpha_{t}^{n},
$$

where $\lambda_{1}$ is a nonzero constant, $\left|\alpha_{1}\right|>\left|\alpha_{j}\right|$ for $j=2, \ldots, t$, and $G_{n}-\lambda_{1} \alpha_{1}^{n} \neq 0$. Then assuming $x, q>1$ the solutions $q$ of (3) can be bounded by an effectively computable constant which depends on the coefficients and initial values of the recurrence. Kiss [8] proved that, in fact, $q$ is less than a number which is effectively computable in terms of the greatest prime divisor of $E$ and the coefficients and the initial values of the sequence $\left(G_{n}\right)$.

Shorey and Stewart [20] considered recurring sequences $\left(G_{n}\right)$ satisfying (4). Assuming that $P_{2}(n)$ is a nonzero constant, $t=3$ and $\left|\alpha_{1}\right|=\left|\alpha_{2}\right|=$ $\left|\alpha_{3}\right|$, they showed that (3) has only finitely many integral solutions $E, x, q$ and $n$ with the greatest prime divisor of $E$ bounded by a given positive integer $P$, and $|x|>1, q>2$ and $n \geq 0$.

Finkelstein [6], Williams [27] and Steiner [24] proved that 1,2 and 5 are the only Fibonacci numbers of the form $x^{2}+1$. Finkelstein [7] established a similar result for Lucas numbers. Stewart [25] and Shorey and Stewart [20] investigated the equation

$$
G_{n}=x^{q}+c,
$$

where $c \in \mathbb{Z}$ and $\left(G_{n}\right)$ is a simple, nondegenerate second order recurring sequence of rational integers. Assuming $\left|A_{2}\right|=1$, they showed for the in- 
tegral solutions of (5) that the maximum of $n \geq 0,|x|>1$ and $q \geq 3$ is less than an effectively computable constant depending on $c$, the coefficients and the initial values of the recurrence. In the case $q=2$ they obtained a similar result under additional technical conditions. Furthermore, Shorey and Stewart [20] proved that if $\alpha_{1}$ and $\alpha_{2}$ are multiplicatively independent with one root inside the unit circle, then (5) has only finitely many solutions in integers $n, x$ and $q$ with $n \geq 0,|x|>1$ and $q>2$.

Nemes and Pethö [11], [12] studied the more general equation

$$
G_{n}=E x^{q}+T(x),
$$

where $T(x)$ is a polynomial of degree $r$ and of height $H$ with integral coefficients. For fixed $E \in \mathbb{Z}$ and $T$ they established bounds for the integral solutions $n, q, x$ with $|x|, q>1$. Let $\left(G_{n}\right)$ be defined as in (4) and assume

$$
\left|\alpha_{1}\right|>\left|\alpha_{2}\right|>\left|\alpha_{j}\right| \quad \text { for } j=3, \ldots, t,
$$

with $\alpha_{2} \neq \pm 1$. Nemes and Pethö showed that $q<C_{1}$ provided that $n>C_{2}$ and $r<C_{3} q$, where $C_{1}, C_{2}$ and $C_{3}$ are suitable positive numbers which are effectively computable in terms of $E, H$ and the coefficients and initial values of the recurrence. Nemes and Pethö were also able to show that if $q$ is a fixed integer larger than one and (6) has infinitely many integral solutions $n$ and $x$, then $T(x)$ can be characterized in terms of the Chebyshev polynomials. Kiss [8] and Shorey and Stewart [20] dealt with equation (6) for nondegenerate linear recurring sequences $\left(G_{n}\right)$ of arbitrary order, under condition $(7)$ and the additional assumptions that $E=1$ and $d$ is the degree of $\alpha_{1}$ over $\mathbb{Q}, \alpha_{1}$ and $\alpha_{2}$ are multiplicatively independent and $\alpha_{2} \neq \pm 1$. They showed that there are then only finitely many integers $n, x$ and $q$ with $n \geq 0,|x|>1$ and

$$
q>\max \left(\frac{d \log \left|\alpha_{1}\right|}{\log \left(\left|\alpha_{1}\right| / \max \left(1,\left|\alpha_{2}\right|\right)\right)}, d+r\right)
$$

for which (6) holds.

Recently Corvaja and Zannier [4] considered linear recurrences defined by

$$
G_{n}=a_{1} \alpha_{1}^{n}+\ldots+a_{t} \alpha_{t}^{n},
$$

where $t \geq 2, a_{1}, \ldots, a_{t}$ are nonzero rational numbers, $\alpha_{1}>\ldots>\alpha_{t}>0$ are integers. They used Schmidt's Subspace Theorem [16], [17] to show that for every integer $q \geq 2$ the equation

$$
G_{n}=x^{q}
$$

has only finitely many solutions $(n, x) \in \mathbb{N}^{2}$ assuming that $G_{n}$ is not identically a perfect $q$ th power for any $n$ in a suitable arithmetic progression.

2. Results. Our first main result gives a quantitative version of the above result of Corvaja and Zannier [4]. 
TheOREM 1. Let $\left(G_{n}\right)$ be a linear recurring sequence defined by

$$
G_{n}=a_{1} \alpha_{1}^{n}+\ldots+a_{t} \alpha_{t}^{n},
$$

where $t \geq 2, a_{1}, \ldots, a_{t}$ are nonzero rational numbers, and $\alpha_{1}>\ldots>\alpha_{t}>0$ are integers such that for given $q \geq 2$ there is no $r \in\{0, \ldots, q-1\}$ with $G_{m q+r}$ a perfect qth power for all $m \in \mathbb{N}$. Then the number of solutions $(n, x) \in \mathbb{N}^{2}$ of the equation

$$
G_{n}=x^{q}
$$

is finite and can be bounded above by an explicitly computable number depending on $q, a_{1}, \ldots, a_{t}, \alpha_{1}, \ldots, \alpha_{t}$.

REMARK 1. Corvaja and Zannier [4] showed that (9) is the $q$ th power of an integer for infinitely many $n \in \mathbb{N}$ if and only if there exist integers $r \in\{0, \ldots, q-1\}, b \geq 1$ and

$$
H_{n}=c_{1} \beta_{1}^{n}+\ldots+c_{s} \beta_{s}^{n},
$$

where $c_{1}, \ldots, c_{s}$ are nonzero rational numbers and $\beta_{1}>\ldots>\beta_{s}>0$ are integers as above, such that

$$
G_{n}=b^{n-r} H_{n}^{q} .
$$

In particular, at least one of the functions $m \mapsto G_{m q+r}(r=0, \ldots, q-1)$ is a $q$ th power in the ring of complex functions (with pointwise multiplication) of the form (9), or $G_{n}$ is a perfect $q$ th power for any $n$ in a suitable arithmetic progression.

REMARK 2. Observe that one can effectively determine whether $G_{m q+r}$ is a perfect $q$ th power or not (see again [4]). A sufficient condition is that $\alpha_{1}, \alpha_{2}$ are coprime.

REMARK 3. The example

$$
G_{n}=18^{n}+2 \cdot 6^{n}+2^{n}
$$

shows that the condition in Theorem 1 that $G_{m q+r}$ not be a perfect $q$ th power for every $m$ cannot be removed. Indeed, in this example the coefficients and roots of $G_{n}$ satisfy the conditions of Theorem 1, but

$$
G_{2 m}=\left(18^{m}+2^{m}\right)^{2},
$$

so $G_{2 m}$ is a perfect square for all $m \in \mathbb{N}$.

REMARK 4. The assumption $\alpha_{1}>\ldots>\alpha_{t}>0$ guarantees that the recurring sequence $\left(G_{n}\right)$ is nondegenerate.

REMARK 5. We mention that the proof of Theorem 1 should also work in the case when $\left(G_{n}\right)$ is a linear recurring sequence with algebraic characteristic roots $\alpha_{1}, \ldots, \alpha_{t}$, which are multiplicatively independent and satisfy

$$
\left|\alpha_{1}\right|>\left|\alpha_{i}\right| \quad \forall i=2, \ldots, t,
$$

and with $a_{i} \in \mathbb{Q}\left(\alpha_{1}, \ldots, \alpha_{t}\right)$ for all $i=1, \ldots, t$. 
Our second main result extends Theorem 1 to the situation when also $q$ is considered to be variable.

TheOREM 2. Let $\left(G_{n}\right)$ be a linear recurring sequence defined by

$$
G_{n}=a_{1} \alpha_{1}^{n}+\ldots+a_{t} \alpha_{t}^{n},
$$

where $a_{1}, \ldots, a_{t}(t \geq 3)$ are nonzero rational numbers and $\alpha_{1}>\ldots>\alpha_{t}>0$ are integers such that (for fixed $q \geq 2$ ) there is no $r \in\{0, \ldots, q-1\}$ with $G_{m q+r}$ a perfect qth power for all $m \in \mathbb{N}$. Then the equation

$$
G_{n}=x^{q}
$$

has only finitely many integral solutions $n, x>1, q$. The number of solutions can be bounded by an explicitly computable constant $C$ depending only on the recurrence.

REMARK 6 . The assumption $t \geq 3$ means no loss of generality, because for $t=2$ this theorem is already well known (see [13], [19]).

REMARK 7. Our proof of Theorem 2 depends on an application of the result of Nemes and Pethő [11] mentioned in the introduction.

REMARK 8. By Remark 5 and the theorem in [11] it should also be possible to obtain the following finiteness result: Let $G_{n}$ be the $n$th term of a linear recurrence sequence defined by (9), where $t \geq 3, \alpha_{1}, \ldots, \alpha_{t}$ are multiplicatively independent algebraic numbers with

$$
\left|\alpha_{1}\right|>\left|\alpha_{2}\right|>\left|\alpha_{i}\right|, \quad \forall i=3, \ldots, t,
$$

and $a_{i} \in \mathbb{Q}\left(\alpha_{1}, \ldots, \alpha_{t}\right)$ for all $i=1, \ldots, t$. Assuming that for fixed $q \geq 2$ there is no $r \in\{0, \ldots, q-1\}$ with $G_{m q+r}$ a perfect $q$ th power for all $m \in \mathbb{N}$, the equation

$$
G_{n}=x^{q}
$$

has only finitely many integral solutions $n, x>1, q$.

3. Auxiliary results. Our proof of Theorem 1 depends on a quantitative version of the Subspace Theorem due to J.-H. Evertse [5].

Let $K$ be an algebraic number field. Denote its ring of integers by $O_{K}$ and its collection of places by $M_{K}$. For $v \in M_{K}, x \in K$, we define the absolute value $|x|_{v}$ by

(i) $|x|_{v}=|\sigma(x)|^{1 /[K: \mathbb{Q}]}$ if $v$ corresponds to the embedding $\sigma: K \hookrightarrow \mathbb{R}$;

(ii) $|x|_{v}=|\sigma(x)|^{2 /[K: \mathbb{Q}]}=|\bar{\sigma}(x)|^{2 /[K: \mathbb{Q}]}$ if $v$ corresponds to the pair of conjugate complex embeddings $\sigma, \bar{\sigma}: K \hookrightarrow \mathbb{C}$;

(iii) $|x|_{v}=\left(N_{\wp}\right)^{-\operatorname{ord}_{\wp}(x) /[K: \mathbb{Q}]}$ if $v$ corresponds to the prime ideal $\wp$ of $O_{K}$.

Here $N \wp=\#\left(O_{K} / \wp\right)$ is the norm of $\wp$ and $\operatorname{ord}_{\wp}(x)$ the exponent of $\wp$ in the prime ideal composition of $(x)$, with $\operatorname{ord}_{\wp}(0):=\infty$. In cases (i) or (ii) 
we call $v$ real infinite or complex infinite, respectively; in case (iii) we call $v$ finite. These absolute values satisfy the product formula

$$
\prod_{v \in M_{K}}|x|_{v}=1 \quad \text { for } x \in K^{*}
$$

The height of $\mathbf{x}=\left(x_{1}, \ldots, x_{n}\right) \in K^{n}$ with $\mathbf{x} \neq \mathbf{0}$ is defined as follows: for $v \in M_{K}$ put

$$
|\mathbf{x}|_{v}= \begin{cases}\left(\sum_{i=1}^{n}\left|x_{i}\right|_{v}^{2[K: \mathbb{Q}]}\right)^{1 /(2[K: \mathbb{Q}])} & \text { if } v \text { is real infinite, } \\ \left(\sum_{i=1}^{n}\left|x_{i}\right|_{v}^{[K: \mathbb{Q}]}\right)^{1 /[K: \mathbb{Q}]} & \text { if } v \text { is complex infinite, } \\ \max \left(\left|x_{1}\right|_{v}, \ldots,\left|x_{n}\right|_{v}\right) & \text { if } v \text { is finite }\end{cases}
$$

(note that for infinite places $v,|\cdot|_{v}$ is a power of the Euclidean norm). Now define

$$
\mathcal{H}(\mathbf{x})=\mathcal{H}\left(x_{1}, \ldots, x_{n}\right)=\prod_{v}|\mathbf{x}|_{v}
$$

For a linear form $l(\mathbf{X})=a_{1} X_{1}+\ldots+a_{n} X_{n}$ with algebraic coefficients we define $\mathcal{H}(l):=\mathcal{H}(\mathbf{a})$, where $\mathbf{a}=\left(a_{1}, \ldots, a_{n}\right)$, and if $\mathbf{a} \in K^{n}$ then we put $|l|_{v}=|\mathbf{a}|_{v}$ for $v \in M_{K}$. Further we define the number field $K(l):=$ $K\left(a_{1} / a_{j}, \ldots, a_{n} / a_{j}\right)$ for any $j$ with $a_{j} \neq 0$; this is independent of the choice of $j$.

We are now ready to state Evertse's result [5]. The following notation is used:

- $S$ is a finite set of places on $K$ of cardinality $s$ containing all infinite places;

- $\left\{l_{1 v}, \ldots, l_{n v}\right\}, v \in S$, are linearly independent sets of linear forms in $n$ variables with algebraic coefficients such that

$$
\mathcal{H}\left(l_{i v}\right) \leq H, \quad\left[K\left(l_{i v}\right): K\right] \leq D \quad \text { for } v \in S, i=1, \ldots, n .
$$

For every place $v \in M_{K}$ we choose a continuation of $|\cdot|_{v}$ to the algebraic closure of $K$ and denote it also by $|\cdot|_{v}$.

Theorem 3 (Quantitative Subspace Theorem, Evertse). Let $0<\delta<1$ and for $\mathbf{x} \in K^{n}$ consider the inequality

$$
\prod_{v \in S} \prod_{i=1}^{n} \frac{\left|l_{i v}(\mathbf{x})\right|_{v}}{|\mathbf{x}|_{v}}<\left(\prod_{v \in S}\left|\operatorname{det}\left(l_{1 v}, \ldots, l_{n v}\right)\right|_{v}\right) \cdot \mathcal{H}(\mathbf{x})^{-n-\delta} .
$$

Then the following assertions hold:

(i) There are proper linear subspaces $T_{1}, \ldots, T_{t_{1}}$ of $K^{n}$ with

$$
t_{1} \leq\left(2^{60 n^{2}} \delta^{-7 n}\right)^{s} \log 4 D \cdot \log \log 4 D
$$


such that every solution $\mathbf{x} \in K^{n}$ of (11) satisfying $\mathcal{H}(\mathbf{x}) \geq H$ belongs to $T_{1} \cup \ldots \cup T_{t_{1}}$.

(ii) There are proper linear subspaces $S_{1}, \ldots, S_{t_{2}}$ of $K^{n}$ with

$$
t_{2} \leq\left(150 n^{4} \delta^{-1}\right)^{n s+1}(2+\log \log 2 H)
$$

such that every solution $\mathbf{x} \in K^{n}$ of (11) satisfying $\mathcal{H}(\mathbf{x})<H$ belongs to $S_{1} \cup \ldots \cup S_{t_{2}}$.

We also need the following theorem of W. M. Schmidt [18] concerning the zero multiplicity of a nondegenerate recurring sequence.

Theorem 4 (W. M. Schmidt). Suppose that $\left(G_{n}\right)_{n \in \mathbb{Z}}$ is a nondegenerate linear recurring sequence of complex numbers whose characteristic polynomial has $k$ distinct roots of multiplicity $\leq a$. Then the number of solutions $n \in \mathbb{Z}$ of the equation

$$
G_{n}=0
$$

can be bounded above by

$$
c(k, a)=e^{\left(7 k^{a}\right)^{8 k^{a}}} .
$$

(This number of solutions is called the zero multiplicity of the recurrence.)

In the proof of Theorem 2 we also apply the following result due to A. Baker [1]. Let us first recall the definition of the absolute logarithmic Weil height: For an algebraic number $\beta$ let $P_{\beta}(x)=x^{k}+a_{k-1} x^{k-1}+\ldots+a_{0} \in \mathbb{Z}[x]$ denote the minimal polynomial of $\beta$. Further let $\beta_{1}=\beta, \beta_{2}, \ldots, \beta_{k}$ denote the conjugates of $\beta$. Then we call

$$
h(\beta)=\frac{1}{k} \log \left(\prod_{i=1}^{k} \max \left\{1,\left|\beta_{i}\right|\right\}\right)
$$

the absolute logarithmic Weil height of $\beta$.

TheORem 5 (A. Baker). Let $\alpha_{1}, \ldots, \alpha_{k}$ be algebraic numbers, different from 0 or $1, K=\mathbb{Q}\left(\alpha_{1}, \ldots, \alpha_{k}\right)$, and let $d$ be the degree $[K: \mathbb{Q}]$. For $i=$ $1, \ldots, k$ set

$$
h_{i}=\max \left\{h\left(\alpha_{i}\right), \frac{e\left|\log \alpha_{i}\right|}{d}, \frac{1}{d}\right\} .
$$

Let $b_{1}, \ldots, b_{k} \in \mathbb{Z}, b_{k}>0, \Lambda=b_{1} \log \alpha_{1}+\ldots+b_{k} \log \alpha_{k} \neq 0$ and $B=$ $\max \left\{2,\left|b_{1}\right|, \ldots,\left|b_{k-1}\right|\right\}$. Then

$$
\log |\Lambda|>-C(k) d^{k+2} h_{1} \ldots h_{k} \log \left(C(k) d^{k+2} h_{1} \ldots h_{k-1}\right) \log b_{k}-\frac{B}{b_{k}},
$$

where

$$
C(k)=2^{26 k} k^{3 k}
$$

A proof of this theorem with the given explicit constants can be found in the monograph of Waldschmidt [26, p. 309, Corollary 9.24]. 
Below we have collected some simple lemmas which are needed in our proofs.

Lemma 1. Let $N_{j, k}$ denote the number of formal summands of $\left(a_{1}+\ldots\right.$ $\left.\ldots+a_{k}\right)^{j}$, where $a_{1}, \ldots, a_{k}$ denote formal commuting variables. Then

$$
N_{j, k}=\left(\begin{array}{c}
k+j-1 \\
j
\end{array}\right)
$$

This is well known from combinatorics.

Lemma 2. Let $d$ be a positive integer. Then for the complex function $f(z)=(1+z)^{1 / d}$ we have

$$
\left|f(z)-\sum_{k=0}^{n}\left(\begin{array}{c}
1 / d \\
k
\end{array}\right) z^{k}\right| \leq \frac{1}{d(n+1)(1-|z|)} \cdot|z|^{n+1}
$$

for $z \in \mathbb{C},|z|<1$, where we have chosen the branch of $(1+z)^{1 / d}$ which is holomorphic on $\mathbb{C} \backslash(-\infty,-1]$ and equal to the positive dth root of $1+z$ for $z \in \mathbb{R}, z>-1$.

Proof. It is well known that for $z \in \mathbb{C},|z|<1$ we have

$$
f(z)=\sum_{k=0}^{\infty}\left(\begin{array}{c}
1 / d \\
k
\end{array}\right) z^{k}
$$

From

$$
(n+1)\left|\left(\begin{array}{c}
1 / d \\
n+1
\end{array}\right)\right|=\frac{\frac{1}{d} \cdot\left(1-\frac{1}{d}\right) \cdot \ldots \cdot\left(n-\frac{1}{d}\right)}{1 \cdot \ldots \cdot n}<\frac{1}{d}<1
$$

we obtain

$$
\begin{aligned}
\left|f(z)-\sum_{k=0}^{n}\left(\begin{array}{c}
1 / d \\
k
\end{array}\right) z^{k}\right| & \leq \sum_{k=n+1}^{\infty}\left|\left(\begin{array}{c}
1 / d \\
k
\end{array}\right)\right| \cdot|z|^{k} \\
& \leq \frac{1}{d(n+1)} \sum_{k=n+1}^{\infty}|z|^{k} \\
& =\frac{1}{d(n+1)(1-|z|)} \cdot|z|^{n+1},
\end{aligned}
$$

and therefore the proof is complete.

Lemma 3. Let $a, b \geq 0$ and let $x \in \mathbb{R}$ be the largest solution of $x=$ $a+b \log x$. If $b>e^{2}$ then

$$
x<2(a+b \log b) .
$$

This lemma is due to A. Pethő and B. M. M. de Weger [22]. 
4. Proof of Theorem 1. According to Theorem 4 the number of solutions of ( 8 ) of the form $(n, 0), n \in \mathbb{N}$, can be estimated by

$$
c_{1}(t) \leq e^{(7 t)^{8 t}} .
$$

Therefore we can restrict ourselves to solutions of the form $(n, x) \in \mathbb{N}^{2}$ with $x \neq 0$. These solutions are denoted by $\left(n, x_{n}\right) \in \mathbb{N}^{2}$ with $n \in \Sigma$, where $\Sigma$ is a set of positive integers.

Let us now consider the expansion of the function $f(z)=(1+z)^{1 / q}$ around the origin,

$$
(1+z)^{1 / q}=\sum_{j=0}^{\infty}\left(\begin{array}{c}
1 / q \\
j
\end{array}\right) z^{j} \quad \text { with }|z| \leq 1, z \neq-1 .
$$

We approximate $G_{n}^{1 / q}$ by defining

$$
H_{m}:=\left(a_{1} \alpha_{1}^{r}\right)^{1 / q} \alpha_{1}^{m}\left[1+\sum_{j=1}^{R}\left(\begin{array}{c}
1 / q \\
j
\end{array}\right) \cdot\left(\sum_{i=2}^{t} \frac{a_{i} \alpha_{i}^{m q+r}}{a_{1} \alpha_{1}^{m q+r}}\right)^{j}\right],
$$

where $R \geq 1$ is an integer to be chosen later and where we have set $n=m q+r$ with $n \in \mathbb{N}, r \in\{0, \ldots, q-1\}$. We write

$$
H_{m}=\sum_{i=1}^{h} d_{i}\left(\frac{e_{i}}{b}\right)^{m}
$$

where $d_{i} \in \mathbb{Q}\left(\left(a_{1} \alpha_{1}^{r}\right)^{1 / q}\right)^{*}, e_{i}, b$ are integers, $b>0$, and the $e_{i} / b$ are nonzero distinct rational numbers. Clearly, $H_{m}$ is nondegenerate (the roots are all positive) and we have

$$
\left[\mathbb{Q}\left(\left(a_{1} \alpha_{1}^{r}\right)^{1 / q}\right): \mathbb{Q}\right] \leq q .
$$

By Lemma 1 we obtain

$$
h \leq\left(\begin{array}{c}
R+t-1 \\
R
\end{array}\right)
$$

On the other hand, we have

$$
\left|\sum_{i=2}^{t} \frac{a_{i} \alpha_{i}^{m q+r}}{a_{1} \alpha_{1}^{m q+r}}\right| \leq(t-1) c\left(\frac{\alpha_{2}}{\alpha_{1}}\right)^{m q+r} \leq \frac{1}{2}<1
$$

where

$$
c:=\max \left\{\left|a_{i} / a_{1}\right| \mid i=2, \ldots, t\right\},
$$

if

$$
m \geq \frac{\log 2(t-1) c}{q \log \frac{\alpha_{1}}{\alpha_{2}}} .
$$

Therefore, by Lemma 2 we get, for $m$ large enough, 


$$
\begin{aligned}
& \left|H_{m}-x_{m q+r}\right|=\left|G_{m q+r}^{1 / q}-H_{m}\right| \\
& \leq\left|a_{1}^{1 / q}\right| \cdot \alpha_{1}^{r / q} \cdot \alpha_{1}^{m} \cdot \frac{1}{q(R+1)\left(1-\left|\sum_{i=2}^{t} \frac{a_{i} \alpha_{i}^{m q+r}}{a_{1} \alpha_{1}^{m q+r}}\right|\right)}\left|\sum_{i=2}^{t} \frac{a_{i} \alpha_{i}^{m q+r}}{a_{1} \alpha_{1}^{m q+r}}\right|^{R+1} \\
& \leq\left|a_{1}^{1 / q}\right| \cdot \alpha_{1} \cdot \alpha_{1}^{m} \cdot \frac{2}{q(R+1)}\left[(t-1) c\left(\frac{\alpha_{2}}{\alpha_{1}}\right)^{r}\left(\frac{\alpha_{2}}{\alpha_{1}}\right)^{m q}\right]^{R+1} \\
& \leq\left|a_{1}^{1 / q}\right| \cdot \alpha_{1} \cdot[(t-1) c]^{R+1} \cdot \alpha_{1}^{m} \cdot\left(\frac{\alpha_{2}}{\alpha_{1}}\right)^{m q(R+1)} .
\end{aligned}
$$

Thus we derive

$$
\left|H_{m}-x_{m q+r}\right| \leq c_{2}(R) l_{1}^{m}
$$

where we have set

$$
l_{1}:=\alpha_{1}\left(\frac{\alpha_{2}}{\alpha_{1}}\right)^{q(R+1)} \quad \text { and } \quad c_{2}(R):=\left|a_{1}^{1 / q}\right| \alpha_{1}[(t-1) c]^{R+1} .
$$

Now choose

$$
R>\max \left\{1, \frac{1}{q} \cdot \frac{\log \alpha_{1}}{\log \frac{\alpha_{1}}{\alpha_{2}}}-1\right\}
$$

Then $0<l_{1}<1$. Put

$$
l:=\frac{l_{1}+1}{2} .
$$

Then for $m$ large enough, to be more precise, for

$$
m>\frac{\log c_{2}(R)}{\log \frac{l}{l_{1}}}
$$

we have

$$
c_{2}(R) l_{1}^{m}=c_{2}(R)\left(\frac{l_{1}}{l}\right)^{m} l^{m}<l^{m}
$$

Consequently, we obtain

$$
\left|H_{m}-x_{m q+r}\right|<l^{m} \quad \text { with } 0<l<1,
$$

provided that $R$ satisfies (15) and $m$ satisfies (13) and (16).

Now let $S$ be the set of places of $\mathbb{Q}$ consisting of $\infty$ and all primes dividing some of the $e_{i}$ or $b$. Extend each place in $S$ to $K:=\mathbb{Q}\left(\left(a_{1} \alpha_{1}^{r}\right)^{1 / q}\right)$ in some way, the infinite place being extended so as to coincide with the complex absolute value in the given embedding of $K$ in $\mathbb{C}$. Define the linear forms $L_{i, v}$ for $v \in S$ and $i=1, \ldots, h$ as follows: $L_{0, \infty}:=L:=X_{0}-\sum_{i=1}^{h} d_{i} X_{i}$, $L_{i, \infty}:=X_{i}$ for $i=1, \ldots, h$, while for $v \in S, v \neq \infty$, put $L_{i, v}:=X_{i}$ for $i=1, \ldots, h$. Then $\left\{L_{0, v}, \ldots, L_{h, v}\right\}, v \in S$, are linearly independent sets of 
linear forms in $h+1$ variables with coefficients in $K$. Furthermore we have

$$
\mathcal{H}\left(L_{i, v}\right)= \begin{cases}\mathcal{H}\left(1,-d_{1}, \ldots,-d_{h}\right)=: \widetilde{H} & \text { for } i=0, v=\infty \\ \mathcal{H}(0, \ldots, 0,1,0, \ldots, 0)=1 & \text { else. }\end{cases}
$$

We set $H:=\max \{1, \widetilde{H}\}$. Then it follows $\mathcal{H}\left(L_{i, v}\right) \leq H$, for $v \in S, i=$ $0, \ldots, h$. Hence $\mathbb{Q}\left(L_{i, v}\right)=\mathbb{Q}$ for $v \neq \infty,\left[\mathbb{Q}\left(L_{0, \infty}\right): \mathbb{Q}\right] \leq q$ and therefore

$$
\left[\mathbb{Q}\left(L_{i, v}\right): \mathbb{Q}\right] \leq q \quad \forall v \in S, i=0, \ldots, h .
$$

For $n \in \Sigma$ define the vector $\mathbf{x}_{m}=\left(b^{m} x_{m q+r}, e_{1}^{m}, \ldots, e_{h}^{m}\right) \in \mathbb{Z}^{h+1}$.

From (17) we obtain $\left|L_{0, \infty}\left(\mathbf{x}_{m}\right)\right| \leq(b l)^{m}$. Recall that $S$ includes all primes dividing $b$ and that the $x_{m q+r}$ are integers. Thus by the product formula (10),

$$
\prod_{v \in S \backslash\{\infty\}}\left|L_{0, v}\left(\mathbf{x}_{m}\right)\right|_{v}=\prod_{v \in S \backslash\{\infty\}}\left|b^{m} x_{m q+r}\right|_{v} \leq \prod_{v \in S \backslash\{\infty\}}\left|b^{m}\right|_{v}=b^{-m} .
$$

Moreover, since $S$ also includes the primes dividing the numbers $e_{i}$, the product formula (10) gives

$$
\prod_{v \in S} \prod_{i=1}^{h}\left|L_{i, v}\left(\mathbf{x}_{m}\right)\right|_{v}=\prod_{v \in S} \prod_{i=1}^{h}\left|e_{i}^{m}\right|_{v}=1 .
$$

Thus we obtain

$$
\prod_{v \in S} \prod_{i=0}^{h} \frac{\left|L_{i, v}\left(\mathbf{x}_{m}\right)\right|_{v}}{\left|\mathbf{x}_{m}\right|_{v}} \leq\left(\prod_{v \in S}\left|\mathbf{x}_{m}\right|_{v}\right)^{-h-1} \cdot l^{m} .
$$

Since the coordinates of the vectors $\mathbf{x}_{m}$ are integers we have $\left|\mathbf{x}_{m}\right|_{v} \leq 1$ for $v \in M_{\mathbb{Q}} \backslash\{\infty\}$. Further, we have

$$
\left|\mathbf{x}_{m}\right|_{\infty} \leq A^{m}
$$

for some real $A$ independent of $m$. Indeed, we have

$$
\left|x_{m q+r}\right| \leq\left|x_{m q+r}-H_{m}\right|+\left|H_{m}\right| \leq l^{m}+h \widetilde{c} a^{m} \leq 1+h \widetilde{c} a^{m} \leq \widetilde{a}^{m}
$$

with

$$
\widetilde{c}:=\max \left\{\left|d_{i}\right| \mid i=1, \ldots, h\right\}, \quad a:=\max \left\{\left|e_{i} / b\right| \mid i=1, \ldots, h\right\},
$$

and $\widetilde{a}:=(1+h \widetilde{c})(1+a)$. Hence

$$
\left|\mathbf{x}_{m}\right|_{\infty}=\left(\left|b^{m} x_{m q+r}\right|^{2}+\sum_{i=1}^{h}\left|e_{i}\right|^{2}\right)^{1 / 2} \leq\left((b \widetilde{a})^{2 m}+h(b a)^{2 m}\right)^{1 / 2} \leq A^{m}
$$

with $A:=(h+1) \widetilde{a} b$. It follows that

$$
\mathcal{H}\left(\mathbf{x}_{m}\right)=\prod_{v \in M_{\mathbb{Q}}}\left|\mathbf{x}_{m}\right|_{v} \leq \prod_{v \in S}\left|\mathbf{x}_{m}\right|_{v} \leq\left|\mathbf{x}_{m}\right|_{\infty} \leq A^{m} .
$$


Lastly we have

which yields

$$
\operatorname{det}\left(L_{0, v}, \ldots, L_{h, v}\right)=\left|\begin{array}{ccccc}
1 & 0 & 0 & \ldots & 0 \\
* & 1 & 0 & \ldots & 0 \\
* & 0 & 1 & \ldots & 0 \\
\vdots & \vdots & \vdots & & \vdots \\
* & 0 & 0 & \ldots & 1
\end{array}\right|=1
$$

$$
\left|\operatorname{det}\left(L_{0, v}, \ldots, L_{h, v}\right)\right|_{v}=1 \quad \forall v \in S .
$$

Combining our estimates we get

$$
\prod_{v \in S} \prod_{i=0}^{h} \frac{\left|L_{i, v}\left(\mathbf{x}_{m}\right)\right|_{v}}{\left|\mathbf{x}_{m}\right|_{v}}<\left(\prod_{v \in S}\left|\operatorname{det}\left(L_{0, v}, \ldots, L_{h, v}\right)\right|_{v}\right) \mathcal{H}\left(\mathbf{x}_{m}\right)^{-h-1-\delta}
$$

for all $m$ with (16), provided that $\delta<\log (1 / l) / \log A$. By Theorem 3 there exist finitely many nonzero rational linear forms $\Lambda_{1}\left(X_{0}, \ldots, X_{h}\right), \ldots$ $\ldots, \Lambda_{g}\left(X_{0}, \ldots, X_{h}\right)$ with

$$
\begin{aligned}
g \leq & \left(2^{60(h+1)^{2}} \delta^{-7(h+1)}\right)^{s} \log 4 q \cdot \log \log 4 q \\
& +\left(150(h+1)^{4} \delta^{-1}\right)^{(h+1) s+1}(2+\log \log 2 H),
\end{aligned}
$$

such that each vector $\mathbf{x}_{m}$ is a zero of some $\Lambda_{j}$.

Suppose first $\Lambda_{j}$ does not depend on $X_{0}$. Then, if $\Lambda_{j}\left(\mathbf{x}_{m}\right)=0$, we have a nontrivial relation

$$
\sum_{i=1}^{h} u_{i}\left(\frac{e_{i}}{b}\right)^{m}=0, \quad u_{i} \in \mathbb{Q}, i=1, \ldots, h .
$$

By Theorem 4 this can hold for at most a finite number of $m$. More precisely, the number of solutions $m$ can be estimated by

$$
c_{1}(h)=e^{(7 h)^{8 h}}
$$

since $\left(H_{m}\right)$ is nondegenerate.

Suppose that $\Lambda_{j}$ depends on $X_{0}$ and that $\Lambda_{j}\left(\mathbf{x}_{m}\right)=0$. Then we have

$$
\sum_{i=1}^{h} v_{i}\left(\frac{e_{i}}{b}\right)^{m}=x_{m q+r}, \quad v_{i} \in \mathbb{Q}, i=1, \ldots, h .
$$

Set

$$
U_{m}:=\sum_{i=1}^{h} v_{i}\left(\frac{e_{i}}{b}\right)^{m}
$$

then $U_{m}$ is a nondegenerate, simple recurring sequence and we obtain

$$
U_{m}^{q}=x_{m q+r}^{q}=G_{m q+r}
$$


Hence

$$
V_{m}:=\left(\sum_{i=1}^{h} v_{i}\left(\frac{e_{i}}{b}\right)^{m}\right)^{q}-\sum_{i=1}^{t} a_{i} \alpha_{i}^{r}\left(\alpha_{i}^{q}\right)^{m}
$$

has the form

$$
V_{m}=\sum_{i=1}^{p} b_{i} \beta_{i}^{m}
$$

with $b_{i} \in \mathbb{Q}, \beta_{i} \in \mathbb{Q}^{+}, i=1, \ldots, p$. Therefore $V_{m}$ is a nondegenerate, simple recurring sequence, and we conclude by Lemma 1 that

$$
p \leq t+\left(\begin{array}{c}
h+q-1 \\
q
\end{array}\right) \leq t+\left(\begin{array}{c}
\left(\begin{array}{c}
R+t-1 \\
t
\end{array}\right)+q-1 \\
q
\end{array}\right) .
$$

Observe that by our assumptions $V_{m}=0$ does not hold for every $m$ hence an $i$ with $b_{i} \neq 0$ exists. Again by Theorem 4 we can bound the number of solutions of (18) by

$$
c_{1}(p)=e^{(7 p)^{8 p}} .
$$

Therefore the number of solutions of (8) can be estimated by

$$
\begin{aligned}
\widetilde{C}(q):= & e^{(7 t)^{8 t}}+\frac{\log 2(t-1) c}{\log \frac{\alpha_{1}}{\alpha_{2}}}+q\left[\left\{\left(2^{60(\widetilde{h}+1)^{2}} \delta^{-7(\widetilde{h}+1)}\right)^{s}\right.\right. \\
& \left.\times \log 4 q \cdot \log \log 4 q+\left(150(\widetilde{h}+1)^{4} \delta^{-1}\right)^{(\widetilde{h}+1) s+1}(2+\log \log 2 H)\right\} \\
& \left.\times\left\{e^{(7 \widetilde{h})^{8 \tilde{h}}}+e^{(7 \widetilde{p})^{8 \tilde{p}}}\right\}+\frac{\log c_{2}(R)}{\log \frac{l}{l_{1}}}\right],
\end{aligned}
$$

where

$$
\begin{aligned}
& \widetilde{h}=\left(\begin{array}{c}
R+t-1 \\
R
\end{array}\right), \quad \widetilde{p}=\left(\begin{array}{c}
\tilde{h}+q-1 \\
q
\end{array}\right)+t, \\
& H=\max \left\{1, \mathcal{H}\left(1,-d_{1}, \ldots,-d_{h}\right)\right\}, \quad s=|S|, \\
& c_{2}(R)=\left|a_{1}^{1 / q}\right| \alpha_{1}[(t-1) c]^{R+1}, \quad \delta<\log (1 / l) / \log A,
\end{aligned}
$$

and $l$ is as in (17).

5. Proof of Theorem 2. Assume that $n, x>1, q \geq 2$ is a solution of (8) and write

$$
x^{q}=G_{n}=a_{1} \alpha_{1}^{n}+B(n) .
$$

We distinguish two cases:

CASE 1: $B(n)=0$. Here we get

$$
|B(n)|=\left|a_{2} \alpha_{2}^{n}\left(1+\sum_{i=3}^{t} \frac{a_{i}}{a_{2}}\left(\frac{\alpha_{i}}{\alpha_{2}}\right)^{n}\right)\right| \geq\left|a_{2}\right| \alpha_{2}^{n}|1-| \sum_{i=3}^{t} \frac{a_{i}}{a_{2}}\left(\frac{\alpha_{i}}{\alpha_{2}}\right)^{n}||>0
$$


since

$$
\begin{aligned}
\left|\sum_{i=3}^{t} \frac{a_{i}}{a_{2}}\left(\frac{\alpha_{i}}{\alpha_{2}}\right)^{n}\right| & \leq \max \left\{\left|\frac{a_{i}}{a_{2}}\right| \mid i=3, \ldots, t\right\} \cdot(t-2)\left(\frac{\alpha_{3}}{\alpha_{2}}\right)^{n} \\
& \leq t c\left|a_{2}\right|^{-1}\left(\frac{\alpha_{3}}{\alpha_{2}}\right)^{n}<1,
\end{aligned}
$$

where $c=\max \left\{\left|a_{i}\right| \mid i=1, \ldots, t\right\}$, whenever

$$
n>\frac{\log \left(t c\left|a_{2}\right|^{-1}\right)}{\log \frac{\alpha_{2}}{\alpha_{3}}}=: n_{1} \text {. }
$$

Therefore $n \leq n_{1}$ must hold and we deduce from $a_{1} \alpha_{1}^{n}=x^{q}$ and $x \geq 2$ that

$$
q=\frac{\log \left(\left|a_{1}\right| \alpha_{1}^{n}\right)}{\log x} \leq \frac{\log \left(c \alpha_{1}^{n_{1}}\right)}{\log 2} .
$$

CASE 2: $B(n) \neq 0$. In this case we first set

$$
\delta:=\frac{1}{2}\left(1-\frac{\log \alpha_{2}}{\log \alpha_{1}}\right) .
$$

Then we get

$$
|B(n)| \leq t c \alpha_{2}^{n}<\frac{1}{2} \alpha_{1}^{n(1-\delta)}
$$

if

$$
n>\frac{2 \log (2 c t)}{\log \frac{\alpha_{1}}{\alpha_{2}}}=: n_{2} \text {. }
$$

Further

$$
\frac{x^{q}}{a_{1} \alpha_{1}^{n}}=1+\frac{B(n)}{a_{1} \alpha_{1}^{n}}
$$

so

$$
1-\left(\left|a_{1}\right| \alpha_{1}^{\delta n}\right)^{-1} \leq\left|a_{1}\right|^{-1} \alpha_{1}^{-n} x^{q} \leq 1+\left(\left|a_{1}\right| \alpha_{1}^{\delta n}\right)^{-1},
$$

where we have used the fact that $\left(\left|a_{1}\right| \alpha_{1}^{\delta n}\right)^{-1}<1 / 2$ if

$$
n>\frac{\log \left(2\left|a_{1}\right|^{-1}\right)}{\delta \log \alpha_{1}}=: n_{3}
$$

Taking logarithms and using the inequalities

$$
|\log (1+x)| \leq x \quad \text { and } \quad|\log (1-x)| \leq 2 x \quad \text { for } 0 \leq x<1 / 2,
$$

from (20) we derive

$$
-2\left|a_{1}\right|^{-1} \alpha_{1}^{-\delta n} \leq-\log \left|a_{1}\right|-n \log \alpha_{1}+q \log x \leq 2\left|a_{1}\right|^{-1} \alpha_{1}^{-\delta n} .
$$

Thus

$$
|-\log | a_{1}\left|-n \log \alpha_{1}+q \log x\right| \leq 2\left|a_{1}\right|^{-1} \alpha_{1}^{-\delta n} .
$$


Put $\Lambda=-\log \left|a_{1}\right|-n \log \alpha_{1}+q \log x$. From (19) and the fact that $B(n) \neq 0$, we get $\Lambda \neq 0$. Thus we can employ Theorem 5 to obtain, for $n \geq 2$,

$$
|\Lambda|>\exp \left\{-C(3) h_{1} h_{2} \log \left(C(3) h_{1} h_{2}\right) e \log x \log q-\frac{n}{q}\right\},
$$

where $C(3)=2^{78} 3^{9}$ and

$$
\begin{aligned}
& h_{1}=\max \left\{h\left(\left|a_{1}\right|^{-1}\right), e|\log | a_{1}||, 1\right\}, \\
& h_{2}=\max \left\{h\left(\alpha_{1}\right), e \log \alpha_{1}, 1\right\}=e \log \alpha_{1} .
\end{aligned}
$$

Set

$$
c_{3}:=C(3) h_{1} h_{2} \log \left(C(3) h_{1} h_{2}\right) e .
$$

A comparison of (21) and (22) reveals that

$$
-c_{3} \log q \log x-\frac{n}{q}<\log \left(2\left|a_{1}\right|^{-1}\right)-n \delta \log \alpha_{1} .
$$

However, for $n>\max \left\{n_{1}, n_{2}\right\}$,

$$
\frac{1}{2}\left|a_{1}\right| \alpha_{1}^{n} \leq\left|a_{1}\right| \alpha_{1}^{n}-|B(n)| \leq x^{q} \leq\left|a_{1}\right| \alpha_{1}^{n}+|B(n)| \leq c t \alpha_{1}^{n} .
$$

Thus, for

$$
n>\max \left\{\frac{\log c t}{\log \alpha_{1}}, \frac{2 \log \left(2\left|a_{1}\right|^{-1}\right)}{\log \alpha_{1}}\right\}=: n_{4}
$$

we obtain

$$
\frac{\log \alpha_{1}}{2} n<q \log x<2 \log \alpha_{1} n .
$$

If we write this as

$$
\frac{n}{q}<\frac{2 \log x}{\log \alpha_{1}}, \quad \frac{\log x}{2 \log \alpha_{1}} q<n,
$$

then (23) can be reformulated as

$$
q<\frac{2 \log \left(\frac{1}{2}\left|a_{1}\right|^{-1}\right)}{\delta \log 2}+\frac{4}{\delta \log \alpha_{1}}+\frac{2 c_{3}}{\delta} \log q .
$$

Thus by Lemma 3,

$$
q<2\left(\frac{2 \log \left(\frac{1}{2}\left|a_{1}\right|^{-1}\right)}{\delta \log 2}+\frac{4}{\delta \log \alpha_{1}}+\frac{2 c_{3}}{\delta} \log \left(\frac{2 c_{3}}{\delta}\right)\right)=: C_{1}
$$

if $n>\max \left\{2, n_{2}, n_{3}, n_{4}\right\}=: n_{5}$. Otherwise, we have

$$
q \leq \frac{\log \left(c t \alpha_{1}^{n_{5}}\right)}{\log 2} .
$$

Altogether we derive

$$
q \leq \max \left\{\frac{\log \left(c t \alpha_{1}^{\bar{c}}\right)}{\log 2}, C_{1}(q)\right\}=: C,
$$


where $\bar{c}:=\max \left\{2, n_{1}, n_{2}, n_{3}, n_{4}\right\}$. For the number of solutions $n, x>1, q \geq 2$ of (8) we finally obtain the upper bound

$$
\sum_{q=2}^{C} \widetilde{C}(q),
$$

and therefore the proof is finished.

Acknowledgements. The authors are most grateful to Professor U. Zannier for several helpful remarks on a previous draft of the present paper. In particular, they are pleased to thank him for his help with handling some exceptional hyperplanes in the proof of Theorem 1. Furthermore, the authors express their thanks to an anonymous referee for various suggestions improving the paper.

\section{References}

[1] A. Baker, A sharpening of the bounds for linear forms in logarithms II, Acta Arith. 24 (1973), 33-36.

[2] J. H. E. Cohn, On square Fibonacci numbers, J. London Math. Soc. 39 (1964), $537-540$.

[3] - Lucas and Fibonacci numbers and some diophantine equations, Proc. Glasgow Math. Assoc. 7 (1965), 24-28.

[4] P. Corvaja and U. Zannier, Diophantine equations with power sums and universal Hilbert sets, Indag. Math. (N.S.) 9 (1998), 317-332.

[5] J. H. Evertse, An improvement of the quantitative subspace theorem, Compos. Math. 101 (1996), 225-311.

[6] R. Finkelstein, On Fibonacci numbers which are one more than a square, J. Reine Angew. Math. 262/263 (1973), 171-178.

[7] -, On Lucas numbers which are one more than a square, Fibonacci Quart. 13 (1975), 340-342.

[8] P. Kiss, Differences of the terms of linear recurrences, Studia Sci. Math. Hungar. 20 (1985), 285-293.

[9] J. C. Lagarias and D. P. Weisser, Fibonacci and Lucas cubes, Fibonacci Quart. 19 (1981), 39-43.

[10] H. London and R. Finkelstein, On Fibonacci and Lucas numbers which are perfect powers, ibid. 7 (1969), 476-481, 487. Corr. 8 (1970), 248.

[11] I. Nemes and A. Pethő, Polynomial values in linear recurrences, Publ. Math. Debrecen 31 (1984), 229-233.

[12] - - - Polynomial values in linear recurrences II, J. Number Theory 24 (1986), $47-53$.

[13] A. Pethő, Perfect powers in second order linear recurrences, ibid. 15 (1982), 5-13.

[14] -, Perfect powers in second order recurrences, in: Topics in Classical Number Theory (Budapest, 1981), Colloq. Math. Soc. János Bolyai 34, North-Holland, Amsterdam, 1984, 1217-1227.

[15] - Full cubes in the Fibonacci sequence, Publ. Math. Debrecen 30 (1983), 117-127.

[16] W. M. Schmidt, Diophantine Approximation, Lecture Notes in Math. 785, Springer, 1980. 
[17] W. M. Schmidt, Diophantine Approximations and Diophantine Equations, Lecture Notes in Math. 1467, Springer, 1991.

[18] - , The zero multiplicity of linear recurrence sequences, Acta Math. 182 (1999), 243-282.

[19] T. N. Shorey and C. L. Stewart, On the Diophantine equation $a x^{2 t}+b x^{t} y+c y^{2}=d$ and pure powers in recurrence sequences, Math. Scand. 52 (1983), 24-36.

[20] - - - Pure powers in recurrence sequences and some related Diophantine equations, J. Number Theory 27 (1987), 324-352.

[21] T. N. Shorey and R. Tijdeman, Exponential Diophantine Equations, Cambridge Univ. Press, Cambridge, 1986.

[22] N. P. Smart, The Algorithmic Resolution of Diophantine Equations, Cambridge Univ. Press, Cambridge, 1998.

[23] R. Steiner, On nth powers in the Lucas and Fibonacci series, Fibonacci Quart. 16 (1978), 451-458.

[24] - On Fibonacci numbers of the form $x^{2}+1$, in: A Collection of Manuscripts Related to the Fibonacci Sequence, Fibonacci Association, Santa Clara, CA, 1980, 208-210.

[25] C. L. Stewart, On some Diophantine equations and related linear recurrence sequences, Sém. Théor. Nombres, Paris, 1980-1981, Birkhäuser, Boston, 1982, $317-$ 321.

[26] M. Waldschmidt, Diophantine Approximation on Linear Algebraic Groups, Springer, Berlin, 2000.

[27] H. C. Williams, On Fibonacci numbers of the form $k^{2}+1$, Fibonacci Quart. 13 (1975), 213-214.

[28] O. Wyler, Squares in the Fibonacci series, Amer. Math. Monthly 71 (1964), 220-222.

Institut für Mathematik

TU Graz

Steyrergasse 30

A-8010 Graz, Austria

E-mail: clemens.fuchs@tugraz.at

tichy@tugraz.at

Received on 28.5.2001

and in revised form on 10.12.2001 